Orthopäde $2009 \cdot 38: 590$

DOI 10.1007/s00132-009-1447-1

Online publiziert: 1. Juli 2009

(c) Springer Medizin Verlag 2009

V. Ewerbec

Abteilung Orthopädie I, Orthopädische Universitätsklinik, Heidelberg

\title{
Von Routineeingriffen und seltenen Fällen
}

\section{Neueste Originalarbeiten und Kasuistiken aus der Orthopädie}

broplastie bringt Ordnungsfaktoren in einen expandierenden klinischen Bereich, zu dem gelegentlich bereits der Begriff „Modeeingriff“ zu hören war. Die klinisch orientierte Originalarbeit zu den Behandlungsergebnissen infizierter Duokopfprothesen bringt klare, differenzierte Hinweise zur Vorgehensweise. Und schließlich ermöglichen die Resultate der Arbeitsgruppe aus der Berliner Charité eine bessere Reproduzierbarkeit von Angaben zum Patellahöhenstand nach Kniegelenkendoprothesenimplantation und somit besser vergleichbare Qualitätsindikatoren.

Die Kasuistiken liefern uns in diesem Heft neben einem interessanten Fallbericht über eine onkologische Rarität drei klinisch bedeutende Warnhinweise mit einem „roten Ausrufungszeichen“. Das Schädigungspotential eines Hochfrequenzmessers für ein metallisches Hüftgelenkimplantat mit der Möglichkeit des Implantatbruches ist sicherlich bisher nicht jedem Kollegen geläufig. Auf die übersehene Ruptur des hinteren Kreuzbandes bei vorliegender vorderer Kreuzbandruptur wird zu Recht in einer weiteren Kasuistik eindringlich hingewiesen. Die letzte Kasuistik schließlich weist darauf hin, dass auch sog. „Routineeingriffe“, wie der endoprothetische Ersatz des Hüftgelenks, tödliche Komplikationsmöglichkeiten beinhalten kann. Der Verwendung des Begriffs „Routineeingriff“ muss nicht nur aus diesem Grunde nachdrücklich entgegen getreten werden. Auch Eingriffe, die häufig durchgeführt werden, bedür- fen der sorgfältigsten Planung und der Durchführung in Kenntnis aller Komplikationsmöglichkeiten, einschließlich der Fähigkeit, diese zu beherrschen.

Sie sehen, liebe Kolleginnen und Kollegen, auch mit den Originalia und den Kasuistiken steht „Der Orthopäde“ mitten im Leben. So soll es bleiben.

In diesem Sinne verbleibe ich mit herzlichen kollegialen Grüßen

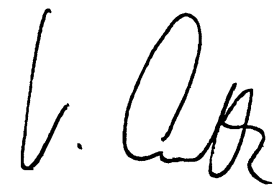

V. Ewerbeck

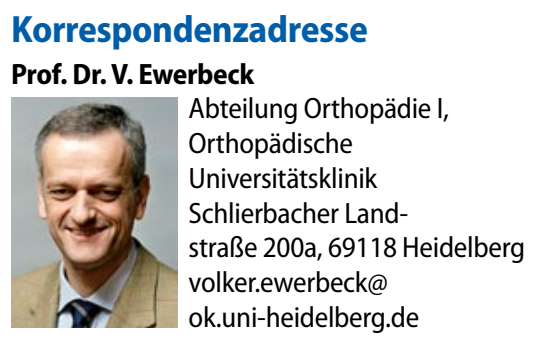

